\title{
Median Stable Matchings in Two-Sided Markets
}

\author{
Peter Chen, Michael Egesdal, Marek Pycia, and M. Bumin Yenmez*
}

Febuary 2016

\begin{abstract}
We show that generalized median stable matchings exist in many-to-many matching markets when contracts are strong substitutes and satisfy the law of aggregate demand.

Keywords: median stable matchings, many-to-one matching with wages, many-to-many matching, matching with contracts, strong substitutability, the law of aggregate demand.
\end{abstract}

\section{Introduction}

We consider a general matching model: there are two sides, such as firms and workers. Each agent can sign a set of contracts with agents on the other side of the market, and each agent has strict preferences over sets of contracts. Each contract specifies a firm, a worker, and the terms of matching between these two agents; they can involve many components such as wages, benefits, etc. The prominent solution concept in such matching markets is stability. If a matching is stable, then each agent is willing

*This paper subsumes some of the results in Chen et al. (2014). Chen is affiliated with Booth School of Business, University of Chicago; Egesdal is affiliated with Harvard University; Pycia is affiliated with UCLA; Yenmez is affiliated with Tepper School of Business, Carnegie Mellon University. Emails: peter_chen@chicagobooth.edu, egesdal@fas.harvard.edu, pycia@ucla.edu, and byenmez@andrew.cmu.edu. For useful comments we would like to thank Federico Echenique, Parag Pathak, and Peng Wang. Yenmez gratefully acknowledges financial support from National Science Foundation grant SES-1326584.

(C) 2016. This manuscript version is made available under the Elsevier user license http://www.elsevier.com/open-access/userlicense/1.0/ 
to keep all of her contracts and there are no additional contracts that agents would like to sign, possibly by dropping some of their current contracts. ${ }^{1}$

Experiments show that agents who match in a decentralized way in the above setting tend to coordinate on a particular stable matching: the median stable matching (Echenique and Yariv, 2013). This evidence raises the question: Under what conditions do median stable matchings exist? Our Theorems 1 and 2 address this question, as well as a more general question of the existence of generalized median stable matchings that are second best, third best, etc. for agents on the same side of the market. In particular, if there is an odd number $k$ of stable matchings then the median stable matching is the $\frac{k+1}{2}$-th best stable matching for all agents on the chosen side.

The extremal matchings that assign the best stable outcome for one side and the worst for the other have been implemented in practice, for instance, in the National Resident Matching Program (NRMP) to match medical doctors to residency programs and in some school districts to match students to high schools (Roth, 1984; Abdulkadiroğlu and Sönmez, 2003). As far as we know, a median stable matching has not yet been implemented despite the fact that it is a focal matching that a market designer may want to implement: It is attractive since it may be seen as a compromise solution that treats both sides of the market in a symmetric way. Indeed, the above experimental results suggest that this is exactly how median stable matchings are perceived by experiment subjects.

To present our results, let us start with the question of the existence of generalized median stable matchings. Suppose that there are $k$ stable matchings. For each agent, consider all the sets of contracts assigned to this agent in the stable matchings and rank them according to this agent's preference. We study the following questions:

1. Existence of generalized median stable matchings: Is the set of contracts that assigns each worker the $i$-th $(1 \leq i \leq k)$ best stable matching outcome, say $X_{W}^{i}$, a matching, and is it stable?

\footnotetext{
${ }^{1}$ Stable matchings exist when contracts are substitutes (Fleiner, 2003). Contracts are substitutes when a contract that is chosen from a larger set is also chosen from a smaller subset that includes that contract.
} 
2. Polarity: When does $X_{W}^{i}$ correspond to the matching that assigns each firm the $k+1-i$-th best stable matching outcome, say $X_{F}^{k+1-i}$ ?

We show that two properties of agent preferences are crucial in addressing the above questions: strong substitutes and the law of aggregate demand. Contracts are strong substitutes if a contract chosen from a set of contracts is also chosen from any worse set of contracts including that contract (Echenique and Oviedo, 2006). Contracts satisfy the law of aggregate demand if the number of chosen contracts from a larger set is weakly greater than the number of contracts chosen from a smaller set (Hatfield and Milgrom, 2005).

Our existence results, Theorems 1 and 2, are as follows. We show that $X_{W}^{i}$ is a stable matching if contracts are substitutes and satisfy the law of aggregate demand for all agents, and are strong substitutes for workers (Theorem 1). On the other hand, under these conditions, $X_{F}^{k+1-i}$ needs not be stable. However, we show that $X_{F}^{k+1-i}$ is a stable matching and corresponds to $X_{W}^{i}$ if contracts are also strong substitutes for firms (Theorem 2). In particular, we show that if $k$ is odd, the median stable matching outcomes for all agents can be attained simultaneously by choosing $i=(k+1) / 2$.

The existence of generalized median stable matchings has been previously established in some special cases of our setting: one-to-one matching with and without wages (Teo and Sethuraman, 1998; Fleiner, 2002; Schwarz and Yenmez, 2011) and the college admissions model with responsive preferences and without wages (Klaus and Klijn, 2006; Sethuraman et al., 2006). ${ }^{2}$ These prior results are implied by our more general treatment. We also resolve the problem posed by Schwarz and Yenmez (2011) who discuss the substantial challenges involved in trying to address the existence of generalized median stable matchings for many-to-one matching markets with wages, leaving the question open. In addition, our Theorems 1 and 2 go beyond this prior literature by identifying the forces behind the generalized median structure

\footnotetext{
${ }^{2}$ In addition, Klaus and Klijn (2010) study the existence of generalized median stable matchings in the roommates problem, and Schwarz and Yenmez (2011) study the case when there is a continuum of potential wages in addition to the finite case.
} 
and polarity results. ${ }^{3}$

\section{Model}

There are two sets of agents: the set of firms $F$, and the set of workers $W$. The set of all agents is denoted by $A \equiv F \cup W$. Each contract $x$ is bilateral and specifies the relationship between a firm-worker pair. The firm and worker associated with contract $x$ are represented by $x_{F} \in F$ and $x_{W} \in W$, respectively. The set of all contracts is finite and denoted by $X$. For a set of contracts $X^{\prime} \subseteq X, X_{a}^{\prime} \equiv\{x \mid x \in$ $\left.X^{\prime}, a \in\left\{x_{F}, x_{W}\right\}\right\}$ denotes the set of contracts that agent $a$ is associated with. A set of contracts $X^{\prime}$ is feasible if for every firm-worker pair $f, w,\left|X_{f}^{\prime} \cap X_{w}^{\prime}\right| \leq 1$, i.e., each firm-worker pair can sign at most one joint contract. A matching is a feasible set of contracts.

Each agent $a$ is endowed with a strict preference relation, which is a linear order $\succ_{a}$ over feasible sets of contracts that involve agent $a$. Similarly, agent $a$ 's weak preference relation is denoted by $\succeq_{a}$, so for all $Y, Y^{\prime} \subseteq X_{a}, Y \succeq_{a} Y^{\prime}$ if and only if $Y \succ_{a} Y^{\prime}$ or $Y=Y^{\prime}$. Given $\succeq_{a}$, let $C_{a}\left(X^{\prime} \mid \succeq_{a}\right)$ denote agent $a$ 's most preferred subset of contracts involving agent $a$ from set $X^{\prime}$. More formally, $C_{a}\left(X^{\prime} \mid \succeq_{a}\right) \subseteq X_{a}^{\prime}$ and for all $Y_{a} \subseteq X_{a}^{\prime}, C_{a}\left(X^{\prime} \mid \succeq_{a}\right) \succeq_{a} Y_{a}$. By definition, the chosen set is a feasible set of contracts involving agent $a$. To ease notation, we suppress the dependence on $\succeq_{a}$ throughout the paper (when this does not lead to ambiguity) and denote the set of contracts chosen from $X^{\prime} \subseteq X$ by $C_{a}\left(X^{\prime}\right)$. Similarly, for any set of contracts $X^{\prime}$, let $C_{W}\left(X^{\prime}\right) \equiv \cup_{w} C_{w}\left(X^{\prime}\right)$ and $C_{F}\left(X^{\prime}\right) \equiv \cup_{f} C_{f}\left(X^{\prime}\right)$ be the chosen sets of contracts for the set of workers and firms, respectively.

Given agents and their preferences, we would like to find a matching that no set of agents would like to deviate from. This is formalized in the following definition of

\footnotetext{
${ }^{3}$ Our results also show that even when generalized median stable matchings do not exist for all agents, they may still exist for one side of the market if contracts are strong substitutes (or preferences are responsive) for that side. In particular, when workers have unit demand, i.e., for all $w \in W$ and $Y \subseteq X\left|C_{w}(Y)\right| \leq 1$, contracts are strong substitutes for workers automatically and, therefore, worker generalized median stable matchings exist if contracts are substitutes and satisfy the law of aggregate demand for firms.
} 
stability.

Definition 1. Given a profile of preference relations $\succ$, a matching $Y$ is stable if

1. for all a, $C_{a}(Y)=Y_{a}$ (individual rationality) and

2. there does not exist a nonempty set of contracts $Z \nsubseteq Y$ such that for all a, $Z_{a} \subseteq C_{a}(Y \cup Z)$ (no blocking).

Stability for a matching entails two things: Individual rationality requires that each agent is better off by holding all of the contracts assigned rather than rejecting some of them. On the other hand, no blocking states that there is no subset of contracts $Z$ such that every agent $a$ would sign $Z_{a}$ if $Z$ is available to them. This is the standard definition of stability for many-to-many matching with contracts: see Hatfield and Kominers (2014).

We make the following assumptions on agents' preferences in our analysis.

Definition 2. Contracts are substitutes in preferences of agent a if for any sets of contracts $Y, Y^{\prime} \subseteq X$ such that $Y \subseteq Y^{\prime}$ and a contract $x$

$$
x \in C_{a}\left(Y^{\prime} \cup\{x\}\right) \Rightarrow x \in C_{a}(Y \cup\{x\}) .
$$

Contracts are substitutes if a contract that is chosen from a set is still chosen from the set's subset that includes the contract. Substitutability is standard in matching: see Kelso and Crawford (1982). It guarantees the existence of a stable matching in our setup (Fleiner, 2003). ${ }^{4}$

Definition 3. Contracts are strong substitutes in preferences of agent a if for any sets of contracts $Y, Y^{\prime} \subseteq X$ such that $C_{a}\left(Y^{\prime}\right) \succeq_{a} C_{a}(Y)$,

$$
x \in C_{a}\left(Y^{\prime} \cup\{x\}\right) \Rightarrow x \in C_{a}(Y \cup\{x\}) .
$$

\footnotetext{
${ }^{4}$ Aygün and Sönmez (2013) show that substitutability alone does not guarantee the existence of stable matchings when choice rules are taken as primitives and prove that an axiom called irrelevance of rejected contracts is needed for the existence. This axiom is satisfied in our setup since choice rules are constructed using strict preferences over sets of contracts.
} 
Strong substitutability implies substitutability. Roughly, it states that if a contract is added to the two sets and chosen from the better set, then it must also be chosen from the worse set. Echenique and Oviedo (2006) introduced strong substitutability for matching markets without contracts. ${ }^{5}$

Definition 4. Contracts satisfy the law of aggregate demand in preferences of agent a if for all $Y, Y^{\prime} \subseteq X$ such that $Y \subseteq Y^{\prime}$

$$
\left|C_{a}(Y)\right| \leq\left|C_{a}\left(Y^{\prime}\right)\right|
$$

The law of aggregate demand requires that the number of contracts chosen from a set is bigger than the number of contracts chosen from a subset of this set (see Alkan (2002); Alkan and Gale (2003); Fleiner (2003); Hatfield and Milgrom (2005)).

\section{Results}

In this section, we introduce median stable matchings and show that they exist if contracts are strong substitutes and satisfy the law of aggregate demand.

Suppose that $\left\{X^{1}, \ldots, X^{k}\right\}$ is the set of stable matchings. For each agent $a$, consider the set of contracts that agent $a$ signs in these matchings: $\left\{X_{a}^{1}, \ldots, X_{a}^{k}\right\}$. Reorder these sets of contracts according to $\succeq_{a}$ such that $X_{a}^{(1)} \succeq_{a} \ldots \succeq_{a} X_{a}^{(k)}$. Let $X_{F}^{i} \equiv \cup_{f \in F} X_{f}^{(i)}$ and $X_{W}^{i} \equiv \cup_{w \in W} X_{w}^{(i)}$ for $1 \leq i \leq k$. In words, $X_{F}^{i}$ assigns each firm the $i$-th best outcome among all stable matching outcomes and $X_{W}^{i}$ assigns each worker the $i$-th best outcome among all stable matching outcomes. We analyze when these sets of contracts are stable.

Theorem 1. Suppose that contracts are substitutes and satisfy the law of aggregate demand for all agents. Suppose also that contracts are strong substitutes for workers. Then for all $i, X_{W}^{i}$ is a stable matching. Moreover, for any firm $f, X_{W}^{i} \succeq_{f} X_{W}^{j}$ if $i \geq j$.

\footnotetext{
${ }^{5}$ As in Echenique and Oviedo (2006), our results would remain valid if we had the strong substitutes condition require only that for agent $a$ if for any sets of contracts $Y, Y^{\prime} \subseteq X$ such that $C_{a}\left(Y^{\prime}\right)=Y_{a}^{\prime}, C_{a}(Y)=Y_{a}$, and $C_{a}\left(Y^{\prime}\right) \succeq_{a} C_{a}(Y)$, we had $x \in C_{a}\left(Y^{\prime} \cup\{x\}\right)$ then $x \in C_{a}(Y \cup\{x\})$.
} 
Under the conditions of Theorem 1, we call $X_{W}^{i}$ a generalized median stable matching for workers. In addition, $X_{W}^{1}$ is called the worker-optimal stable matching and $X_{W}^{k}$ is the worker-pessimal stable matching. In the symmetric case, when contracts are strong substitutes for firms, we define the generalized median, firm-optimal, and firm-pessimal stable matchings for firms. The worker-optimal and firm-optimal stable matchings still exist when contracts are just substitutes and satisfy the law of aggregate demand for all agents. This follows from the lattice structure shown in Fleiner (2003). However, the other matchings need not be stable when strong substitutability fails (see Chen et al. (2014) for an example).

A direct consequence of this result is that when workers have unit demand generalized median stable matchings exist. This answers the open question posed in Schwarz and Yenmez (2011) about the existence of generalized median stable matchings in many-to-one matching markets when contracts are written over wages when there is a finite set of contracts.

Corollary 1. Suppose that workers have unit demand, i.e., for all $w \in W$ and $Y \subseteq X\left|C_{w}(Y)\right| \leq 1$. Suppose also that contracts are substitutes and satisfy the law of aggregate demand for firms. Then for all $i, X_{W}^{i}$ is a stable matching. Moreover, for any firm $f, X_{W}^{i} \succeq_{f} X_{W}^{j}$ if $i \geq j$.

Next we show that generalized median stable matchings for firms and workers are exactly the same with the polarization of interests property when contracts are also strong substitutes for all firms.

Theorem 2. Suppose that contracts are strong substitutes and satisfy the law of aggregate demand for all agents. Then for all $i, X_{W}^{i}$ and $X_{F}^{i}$ are stable matchings; moreover, $X_{W}^{i}=X_{F}^{k+1-i}$.

As a corollary we can also obtain the same result for the setting with responsive preferences. The definition of responsive preferences (Definition 5.2 in Roth and Sotomayor (1990)) can be naturally extended to the setting with contracts, and our earlier draft Chen et al. (2014) provides the details of the argument.

If contracts are strong substitutes and satisfy the law of aggregate demand, then the generalized median stable matchings for both firms and workers exist. Moreover, 
these matchings are aligned in the following way: the worker-optimal stable matching is the firm-pessimal stable matching, the (2)-nd generalized stable matching for workers is the $(k-1)$-th generalized median stable matching for firms, etc. In particular, when $k$ is odd, there exists a stable matching that assigns all agents their median stable matching outcomes since $X_{F}^{(k+1) / 2}=X_{W}^{(k+1) / 2}$.

The examples in the appendix of Chen et al. (2014) demonstrate that the generalized median stable matchings need not exist if we weaken strong substitutability to substitutability or get rid of the law of aggregate demand.

All the generalized medians are potentially different and the mechanisms that consistently map preference profiles to a selected generalized median stable matching are all different. Furthermore, these mechanisms can be ranked in terms of manipulability using definitions coined by Day and Milgrom (2008), Pathak and Sönmez (2013), and Chen et al. (2015). In particular, as we choose a higher generalized median for one side of the market, we make the mechanism less manipulable for that side, but more manipulable for the other side of the market. Thus, no two such mechanisms is better than another in terms of manipulability when all agents in the market are strategic. See Chen et al. (2014) for the construction of the generalized median mechanisms, and the statements and proofs of these assertions. ${ }^{6}$

\section{Appendix: Proofs}

We provide two lemmas that are used in the proofs of Theorems 1 and 2 .

A stable matching exists once we impose that contracts are substitutes (Fleiner, 2003). Here, we study the structure of the set of stable matchings if, in addition to substitutability, contracts satisfy the law of aggregate demand. In particular, we are interested in when this set is a lattice with respect to the following operators.

Let $Y$ and $Y^{\prime}$ be two sets of contracts. Define the following sets of contracts:

$$
Y \vee_{F} Y^{\prime}=\bigcup_{f} \max _{\succeq_{f}}\left\{Y_{f}, Y_{f}^{\prime}\right\}
$$

\footnotetext{
${ }^{6}$ Chen et al. (2014) refer to these mechanisms as quantile stable mechanisms.
} 
and

$$
Y \wedge_{F} Y^{\prime}=\bigcup_{f} \min _{\succeq_{f}}\left\{Y_{f}, Y_{f}^{\prime}\right\}
$$

Operator $\vee_{F}$ chooses the most preferred set of contracts for each firm. On the other hand, $\wedge_{F}$ chooses the least preferred set of contracts for each firm. Analogously, we define $Y \vee_{W} Y^{\prime}$ and $Y \wedge_{W} Y^{\prime}$ :

$$
Y \vee_{W} Y^{\prime}={ }_{w} \bigcup \max _{\succeq w}\left\{Y_{w}, Y_{w}^{\prime}\right\}
$$

and

$$
Y \wedge_{W} Y^{\prime}={ }_{w} \bigcup \min _{\succeq_{w}}\left\{Y_{w}, Y_{w}^{\prime}\right\}
$$

By definition, all of these operators define a set of contracts but in general they do not have to be stable matchings. We study this question in the following lemma.

Lemma 1. Suppose that contracts are substitutes and satisfy the law of aggregate demand for all agents. Suppose also that contracts are strong substitutes for workers. Then, for any two stable matchings $Y$ and $Y^{\prime}, Y \vee_{W} Y^{\prime}$ and $Y \wedge_{W} Y^{\prime}$ are stable matchings. Moreover, for each firm $f,\left(Y \wedge_{W} Y^{\prime}\right) \succeq_{f} Y, Y^{\prime}$ and $Y, Y^{\prime} \succeq_{f}\left(Y \vee_{W} Y^{\prime}\right)$.

Proof. Let $x \in Y \vee_{W} Y^{\prime}$, so there exists $w$ such that $x \in \max _{\succeq_{w}}\left\{Y_{w}, Y_{w}^{\prime}\right\}$. We want to show that $x \in C_{w}\left(Y \cup Y^{\prime}\right)$. If $x \in Y_{w} \cap Y_{w}^{\prime}$ then the claim follows from the following observation that is implied by Corollary 26 and Equation 38 in Fleiner (2003).

Obervation 1. Suppose that contracts are substitutes and satisfy the law of aggregate demand for all agents. If $Y$ and $Y^{\prime}$ are stable matchings, then $C_{W}\left(Y \cup Y^{\prime}\right)$ and $C_{F}\left(Y \cup Y^{\prime}\right)$ are stable matchings such that

$$
Y \cup Y^{\prime}=C_{F}\left(Y \cup Y^{\prime}\right) \cup C_{W}\left(Y \cup Y^{\prime}\right) \text { and } Y \cap Y^{\prime}=C_{F}\left(Y \cup Y^{\prime}\right) \cap C_{W}\left(Y \cup Y^{\prime}\right) \text {. }
$$

Let us thus suppose $x \in Y_{w}$ and $x \notin Y_{w}^{\prime}$. This implies that $Y_{w} \succeq_{w} Y_{w}^{\prime}$.

Since $Y$ is stable, we have $x \in Y_{w}=C_{w}\left(Y_{w}\right)=C_{w}\left(Y_{w} \cup\{x\}\right)$. Thus $Y_{w} \succeq_{w} Y_{w}^{\prime}$ and strong substitutes imply $x \in C_{w}\left(Y_{w}^{\prime} \cup\{x\}\right)$. Let $f$ be $x_{F}$. If $x \in C_{f}\left(Y_{f}^{\prime} \cup\{x\}\right)$, then $\{x\}$ would block $Y^{\prime}$, which contradicts the stability of $Y^{\prime}$. Therefore, $x \notin C_{f}\left(Y_{f}^{\prime} \cup\{x\}\right)$. 
Substitutability then implies that $x \notin C_{f}\left(Y_{f}^{\prime} \cup Y_{f}\right)$ and $x \notin C_{F}\left(Y^{\prime} \cup Y\right)$. The observation highlighted above then implies $x \in C_{W}\left(Y \cup Y^{\prime}\right)$. Hence $Y \vee_{W} Y^{\prime} \subseteq$ $C_{W}\left(Y \cup Y^{\prime}\right)$.

Since $Y, Y^{\prime}$, and $C_{W}\left(Y^{\prime} \cup Y\right)$ are stable matchings, the rural hospital theorem implies $\left|Y_{w^{\prime}}\right|=\left|Y_{w^{\prime}}^{\prime}\right|=\left|C_{w^{\prime}}\left(Y \cup Y^{\prime}\right)\right|$ for every worker $w^{\prime} .{ }^{7}$ By construction, for every worker $w^{\prime},\left|\left(Y \vee_{W} Y^{\prime}\right)_{w^{\prime}}\right|=\left|Y_{w^{\prime}}\right|=\left|Y_{w^{\prime}}^{\prime}\right|$, so $\left|Y \vee_{W} Y^{\prime}\right|=|Y|=\left|Y^{\prime}\right|=\left|C_{W}\left(Y \cup Y^{\prime}\right)\right|$. The inclusion proven above allows us to conclude that $Y \vee_{W} Y^{\prime}=C_{W}\left(Y \cup Y^{\prime}\right)$. Therefore, $Y \vee_{W} Y^{\prime}$ is a stable matching.

Denote by $\chi$ the indicator function on sets of contracts. By the observation highlighted above, $\chi(Y)+\chi\left(Y^{\prime}\right)=\chi\left(C_{F}\left(Y \cup Y^{\prime}\right)\right)+\chi\left(C_{W}\left(Y \cup Y^{\prime}\right)\right)$ and, by definition, $\chi\left(Y \vee_{W} Y^{\prime}\right)+\chi\left(Y \wedge_{W} Y^{\prime}\right)=\chi(Y)+\chi\left(Y^{\prime}\right)$. We thus get $\chi\left(Y \vee_{W} Y^{\prime}\right)+\chi\left(Y \wedge_{W} Y^{\prime}\right)=$ $\chi\left(C_{F}\left(Y \cup Y^{\prime}\right)\right)+\chi\left(C_{W}\left(Y \cup Y^{\prime}\right)\right)$. Above we have shown that $Y \vee_{W} Y^{\prime}=C_{W}\left(Y \cup Y^{\prime}\right)$, so $\chi\left(Y \vee_{W} Y^{\prime}\right)=\chi\left(C_{W}\left(Y \cup Y^{\prime}\right)\right)$. Therefore, $\chi\left(Y \wedge_{W} Y^{\prime}\right)=\chi\left(C_{F}\left(Y \cup Y^{\prime}\right)\right)$ and thus $Y \wedge_{W} Y^{\prime}=C_{F}\left(Y \cup Y^{\prime}\right)$, so $Y \wedge_{W} Y^{\prime}$ is a stable matching.

The last claim of the lemma now follows similarly to the polarization of interest property established by Echenique and Oviedo (2006) (Theorem 9.8); while they derive the polarization of interests for many-to-many matching markets without contracts, an analogous argument works in the setting with contracts we study.

Next we impose that contracts are strong substitutes for all agents to show that $\vee_{F}=\wedge_{W}$ and $\vee_{W}=\wedge_{F}$.

Lemma 2. Suppose that contracts are strong substitutes and satisfy the law of aggregate demand for all agents. Then for any two stable matchings $Y$ and $Y^{\prime}$, $Y \vee_{F} Y^{\prime}=Y \wedge_{W} Y^{\prime}, Y \wedge_{F} Y^{\prime}=Y \vee_{W} Y^{\prime}$. Moreover, $Y \vee_{F} Y^{\prime}$ and $Y \wedge_{F} Y^{\prime}$ are stable matchings.

Proof. This result follows directly from Lemma 1 above. In the proof of Lemma 1, we have shown that $Y \vee_{W} Y^{\prime}$ and $Y \wedge_{W} Y^{\prime}$ are stable matchings, $Y \vee_{W} Y^{\prime}=C_{W}\left(Y \cup Y^{\prime}\right)$, and $Y \wedge_{W} Y^{\prime}=C_{F}\left(Y \cup Y^{\prime}\right)$, relying on the assumption that contracts are strong

7 The rural hospitals theorem says that any agent signs the same number of contracts in any stable matching when contracts are substitutes and satisfy the law of aggregate demand; in the setting of our lemma it was proved by Fleiner (2003). 
substitutes for workers. Symmetrically, since contracts are strong substitutes for firms, $Y \vee_{F} Y^{\prime}$ and $Y \wedge_{F} Y^{\prime}$ are stable matchings, where $Y \vee_{F} Y^{\prime}=C_{F}\left(Y \cup Y^{\prime}\right)$, and $Y \wedge_{F} Y^{\prime}=C_{W}\left(Y \cup Y^{\prime}\right)$. Therefore, $Y \vee_{W} Y^{\prime}=Y \wedge_{F} Y^{\prime}$ and $Y \wedge_{W} Y^{\prime}=Y \vee_{F} Y^{\prime}$.

From here, the proofs of Theorems 1 and 2 parallel the proofs of Theorem 5.5 in Fleiner (2002), Theorem 3.2 in Klaus and Klijn (2006), and Theorem 1 in Schwarz and Yenmez (2011). They may be found in a previous draft.

\section{References}

AbdulkadiroĞLu, A. And T. Sönmez (2003): "School Choice: A Mechanism Design Approach," American Economic Review, 93, 729-747.

Alkan, A. (2002): "A class of multipartner matching models with a strong lattice structure," Economic Theory, 19, 737-746.

Alkan, A. And D. Gale (2003): "Stable schedule matching under revealed preference," Journal of Economic Theory, 112, 289 - 306.

Aygün, O. And T. Sönmez (2013): "Matching with Contracts: Comment," American Economic Review, 103, 2050-2051.

Chen, P., M. Egesdal, M. Pycia, And M. B. Yenmez (2014): "Quantile Stable Mechanisms," Working Paper.

- (2015): "Manipulability of Stable Mechanisms," Forthcoming in May 2016, American Economic Journal: Microeconomics.

Day, R. And P. Milgrom (2008): "Core-selecting Package Auctions," International Journal of Game Theory, 36, 393-407.

Echenique, F. And J. Oviedo (2006): "A Theory of Stability in Many-to-Many Matching Markets," Theoretical Economics, 1, 233-273.

Echenique, F. AND L. YARIV (2013): “An Experimental Study of Decentralized Matching," Working Paper. 
Fleiner, T. (2002): "Some results on stable matchings and fixed points," Working Paper.

- (2003): "A Fixed-Point Approach to Stable Matchings and Some Applications," Mathematics of Operations Research, 28, 103-126.

Hatfield, J. And P. Milgrom (2005): "Matching with Contracts," American Economic Review, 95, 913-935.

Hatfield, J. W. And S. D. Kominers (2014): "Contract Design and Stability in Manyto-Many Matching," Working Paper.

Kelso, A. S. And V. P. Crawford (1982): "Job Matching, Coalition Formation, and Gross Substitutes," Econometrica, 50, 1483-1504.

Klaus, B. And F. KLiJn (2006): "Median stable matching for college admissions," International Journal of Game Theory, 34, 1-11.

- (2010): "Smith and Rawls share a room: stability and medians," Social Choice and Welfare, 35, 647-667.

Pathak, P. And T. SÖnmez (2013): "School Admissions Reform in Chicago and England: Comparing Mechanisms by Their Vulnerability to Manipulation," American Economic Review, 1031, 80-106.

Roth, A. E. (1984): "The Evolution of the Labor Market for Medical Interns and Residents: A Case Study in Game Theory," Journal of Political Economy, 92, 991-1016.

Roth, A. E. AND M. A. O. Sotomayor (1990): Two-sided matching: a study in gametheoretic modeling and analysis, Cambridge: Econometric Society monographs.

Schwarz, M. And M. B. Yenmez (2011): "Median Stable Matching for Markets with Wages," Journal of Economic Theory, 146, 619-637.

Sethuraman, J., C.-P. Teo, And L. Qian (2006): "Many-to-One Stable Matching: Geometry and Fairness," Mathematics of Operations Research, 31, 581-596.

Teo, C.-P. And J. Sethuraman (1998): "The geometry of fractional stable matchings and its applications," Mathematics of Operations Research, 23, 874-891. 